\title{
Signal conditioning algorithms for enhanced tactical sensor imagery
}

\author{
Klamer Schutte, Dirk-Jan de Lange, and Sebastian P. van den Broek \\ TNO Physics and Electronics Laboratory, P.O. Box 96864, 2509 JG, The Hague, The Netherlands
}

\begin{abstract}
In tactical sensor imagery there always is a need for less noise, higher dynamic range and more resolution. Although recent developments lead to better and better Focal Plane Array (FPA) camera systems, modern infrared FPA camera system are still hindered by non-uniformities, a limited signal-to-noise ratio and a limited spatial resolution. The current availability of fast and inexpensive digital electronics allows the use of advanced real-time signal processing to address the need for better image quality. We will present results of signal-conditioning algorithms, which achieve signifcant better performance with regard to the FPA problems given above. Scene-Based Non-Uniformity Correction (SBNUC) can provide an on-line correction of existing and evolving £xed-pattern noise. Dynamic Super Resolution (DSR) improves the signal-to-noise ratio, while simultaneously improving spatial resolution. The signal-conditioning algorithms can handle camera movements, high temporal noise levels, high £xed-pattern noise levels and large moving objects. The Local Adaptive Contrast Enhancement (LACE) algorithm does effectively compress the 10, 12 or 14 bits dynamic range of the corrected imagery towards a 6 to 8 bits dynamic range for the display system, without the loss of image details. In this process, it aims at keeping all information in the original image visible. We will show that the SBNUC, DSR, mosaic generation, and LACE can be integrated in a very natural way resulting in excellent all-round performance of the signalconditioning suite. We will demonstrate the application of SBNUC, DSR, Mosaicking and LACE for various imaging systems, showing signifcant improvement of the image quality for several imaging conditions.
\end{abstract}

Keywords: non-uniformity correction, noise reduction, super resolution, electronic stabilization, mosaic generation, contrast enhancement

\section{INTRODUCTION}

The speed, size, power consumption and price of current electronics allow the application of advanced algorithms for signal conditioning of modern camera systems. This paper will discuss the Scene Based Non-Uniformity Correction (SBNUC), Dynamic Super Resolution (DSR) and Local Adaptive Contrast Enhancement (LACE) algorithms with their application for noise reduction, resolution enhancement, image sharpening, stabilization, mosaic generation, moving target detection and contrast enhancement.

The signal conditioning techniques are applicable to a wide range of camera systems and application areas. Section 8 gives examples for mid and long wave Infra-Red (IR) cameras, image intensifers and visual light cameras. The application areas for which we provide examples are air-to-ground and ground-to-ground scenarios. The algorithms are usable in all scenarios where there is relative motion between the scene and the camera, and the scene should not be more than slowly varying.

The techniques presented in this paper can be implemented in real-time with current technology, due to their moderate computational needs of less than 100 operations per pixel. The nature of the algorithms allow both Digital Signal Processor (DSP) and Programmable Logic Devices (PLD) based solutions depending on the amount of aexibility required.

\section{NOISE REDUCTION}

For signal conditioning purposes we identify two distinct classes of noise in sensor imagery. The frst class is where the noise is correlated in time, and the other class is where noise is not correlate in time. Typical examples of the frst class are non-uniformities in IR Focal Plane Arrays (FPAs) and non-uniform readout circuits. Typical examples of the second class are readout electronic noise and Poisson noise caused by photon count statistics.

Corresponding author: Klamer Schutte: E-mail: Schutte@fel.tno.nl Phone: +31 703740469 Fax: +31 703740654 


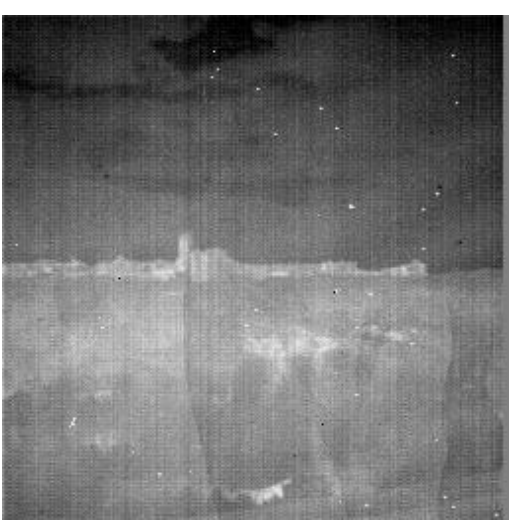

(a)

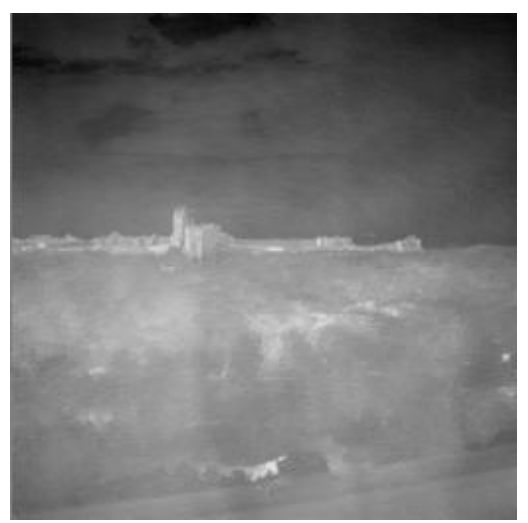

(b)

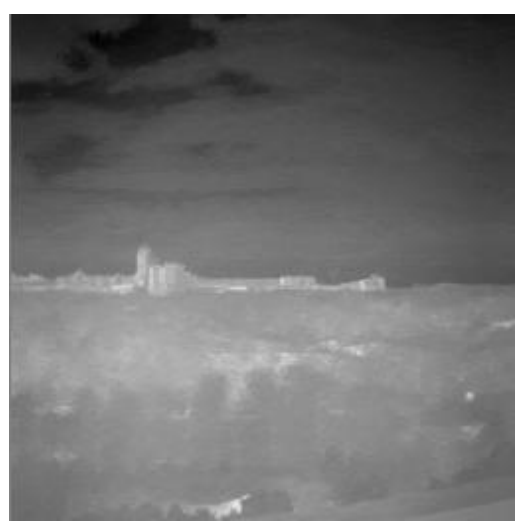

(c)

Figure 1. Decrease by SBNUC of non-uniformity over time. (a) Original InSb image without NUC applied. (b) SBNUC corrected image after 10 frames. (c) SBNUC corrected image after 500 frames. It is clear that the smaller NUC structures are faster corrected than the larger.

\subsection{Non-uniformity correction}

With many manufacturing processes for IR FPAs it is not possible to have completely similar responses for all detector cells. In the remainder of this paper we will address this different response for each cells as non-uniformities. Standard methods of reducing the effect of such non-uniformities include single and multi-point corrections of each detector element's response curve to match the ideal response. Although such approaches are quite successful in reducing the non-uniformities there also are associated problems:

- The response curve is not constant over time. Due to varying FPA temperature and detector aging a one-time correction will not suf£ce over the life-time of the detector.

- To enable regular re-calibration, many IR camera systems are equipped with mechanisms to place reference plates in the optical path. The reference plate should have a uniform temperature. Such a re-calibration introduces an interruption in the service of the camera system. Addition of such a mechanical re-calibration system does introduce extra moving mechanical parts, leading to drawbacks in size, weight, maintenance and costs.

- Single and multi-point corrections only are an approximation of the actual non-uniformity of a detector. This means that after such a correction residual non-uniformity will remain. Multi-point corrections can provide better approximations, but are less suitable in combination with re-calibration.

We propose to use Scene Based Non-Uniformity Correction (SBNUC) which can provide a continuous update to the estimate of the current non-uniformity of each detector cell. SBNUC is based on the assumption of having a slowly (relative to the frame rate) varying scene and (relative) camera movement. The key idea within SBNUC is that within the camera reference frame the scene content will be moving and that the non-uniformities will be semi-static. Accurate motion estimation is used to identify the actual apparent scene movement. This apparent scene movement is used to calculate a motion-corrected difference image. Using the assumption of a slowly varying scene it is obvious that this difference image will contain the current residual non-uniformity (and is cluttered by temporal noise.) Based upon an advanced image formation model, SBNUC does provide the optimal reduction of the non-uniformities over time. The current SBNUC implementation focuses on reduction of the spatial high frequencies of the non-uniformities. This is visualized in £gure 1 , where can be seen that corrections for the high spatial frequency components are faster than the corrections for the low frequency components.

\subsection{Temporal noise reduction}

Under the assumption of slowly varying scenes, temporal noise can easily be identi£ed as fast changes in the imagery. For static cameras and scenes this often is performed by simple averaging over the image sequence. For non-static cameras and/or scenes, smarter noise reduction algorithms are needed. Straightforward implementations using motion estimation, 


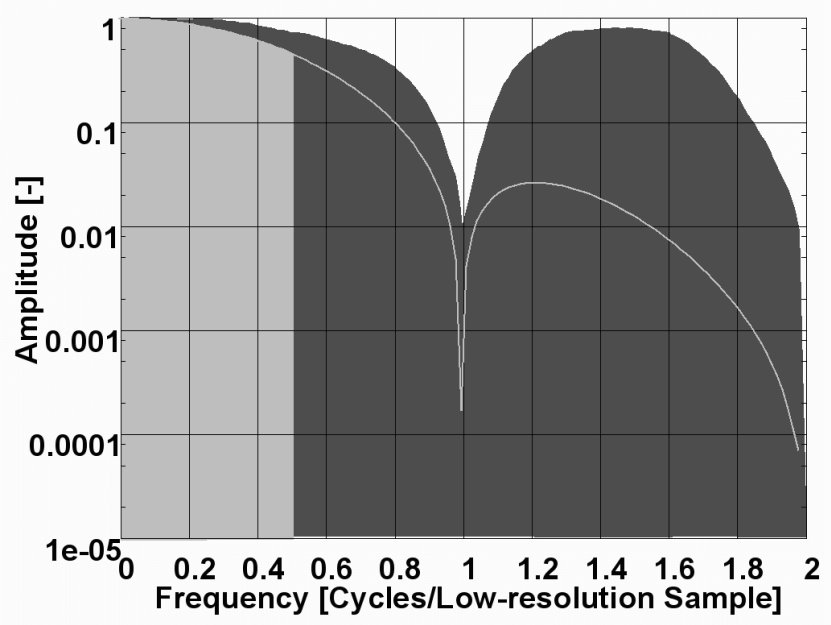

Figure 2. Performance of DSR. The light gray area on the left is the response of a standard aliasing camera system. The gray curve is the response due to optics PSF and fnite sampling element size. The dark gray area is the measured response for DSR-4, which includes resolution enhancement and image sharpening.

motion correction and averaging will suffer from either introduction of aliasing noise or from blurring of the scene content in the motion correction process. ${ }^{8}$ As such, proper implementations of temporal noise reduction need more advanced algorithms, such as described in the next section.

\section{SUPER RESOLUTION}

The term super resolution as used in this paper describes the generation of higher resolution imagery using resolution enhancement and image sharpening techniques.

Earlier work has been reported on separate resolution enhancement and image sharpening. ${ }^{1,2}$ Other work reported on integrated solutions but did not manage (near) real time performance. ${ }^{4,5}$ Our Dynamic Super Resolution (DSR) algorithm aims at simultaneous temporal noise reduction, resolution enhancement and image sharpening. Combination of these functions in a combined algorithm allows a well balanced result.

\subsection{Resolution enhancement}

Resolution enhancement is a process in which the amount of pixels in an image is increased by a factor $n$. We de£ne $n$ such that $n$ times resolution enhancement to a image with $M \times N$ pixels results in an image with $n M \times n N$ pixels.

Resolution enhancement, similar to temporal noise reduction, can be based on motion estimation and motion correction. The disadvantages to straightforward approaches, as mentioned earlier for temporal noise reduction, are even more apparent when applied to resolution enhancement. The enhanced resolution obviously needs a very clear image, which cannot be reached when any blurring of scene content will happen.

\subsection{Image sharpening}

Image sharpening is a process where higher spatial frequencies within an image are enhanced. In general, increasing the system response for higher spatial frequencies can lead to ringing and also will increase the noise. Often a controlling mechanism, such as the Wiener £lter, ${ }^{3}$ is used to limit both ringing and amplifed noise in the image sharpening process.

In the DSR algorithm the temporal noise reduction, motion correction, resolution enhancement and image sharpening functions are matched and integrated. For optimal performance it relies on accurate motion estimation, allowing motion correction with minimal motion artifacts. There is a trade-space between the amount of noise reduction and the amount of high spatial frequencies provided by the DSR algorithm. This allows for high-noise low-light level TV images to apply only noise reduction, while for high-end high-contrast scenes to provide signifcant resolution gain. DSR resolution enhancement and image sharpening performance as measured for a good signal to noise ratio scenario is given in £gure 2.

Proc. of SPIE Vol. 5076 

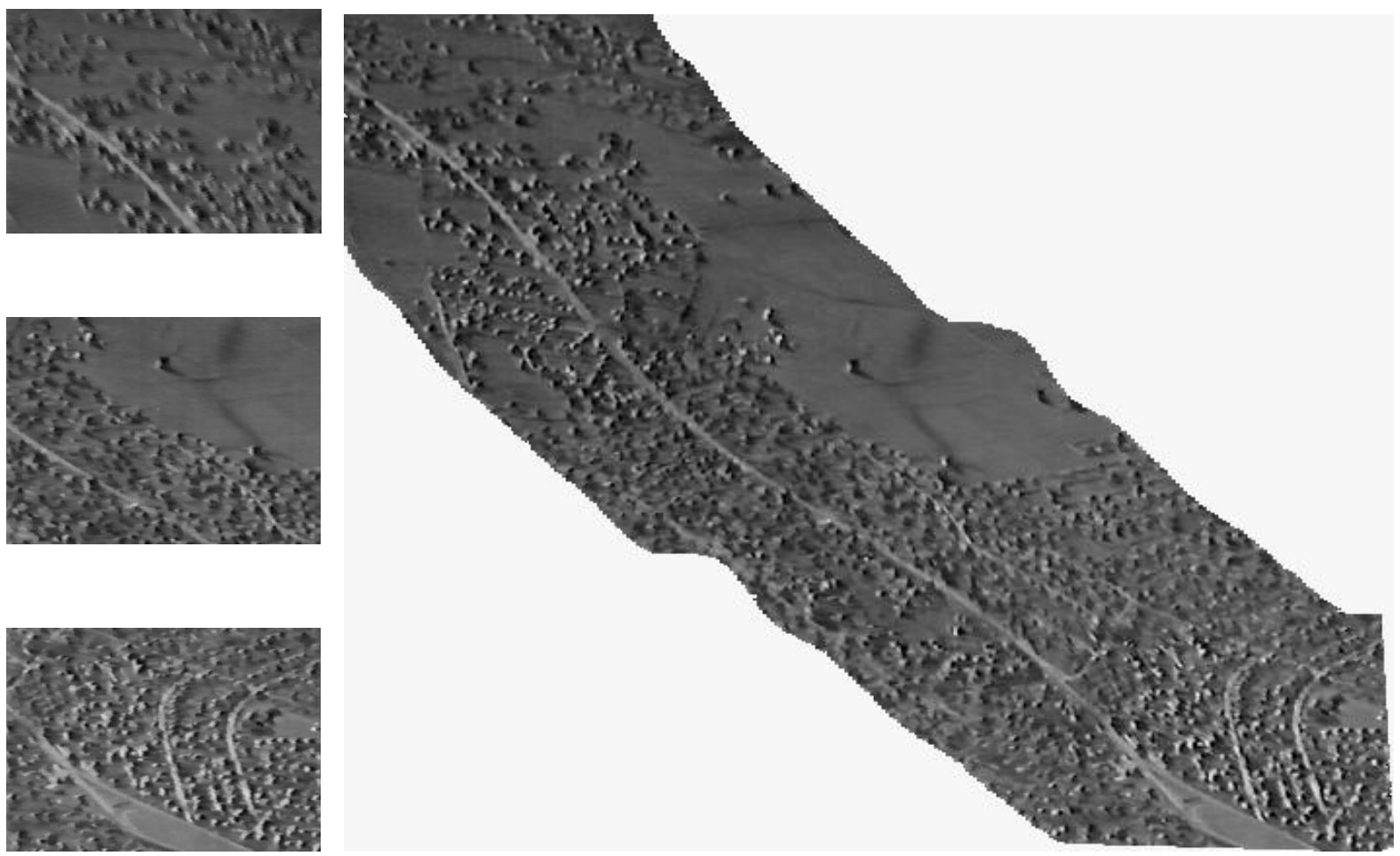

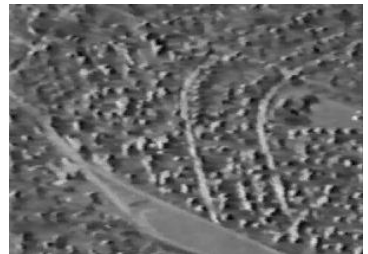

(a)

(b)

Figure 3. Example of mosaicking. (a) Sample images in the sequence. (b) Resulting mosaic.

\section{STABILIZATION AND MOSAIC GENERATION}

Both the SBNUC and DSR algorithm rely on an accurate motion estimation input. This is provided by a Scene Based Motion Estimation (SBME) algorithm. The advantage of an accurate SBME algorithm is that there is no need for (expensive) external motion sensors. Also, since the image information itself is used for motion estimation, application of an SBME algorithm eliminates the possibility of errors due to vibration between the motion sensor and the imaging system.

To achieve accurate SBME algorithm its motion model should match the actual scene motion. Current implemented models include translation-only and af£ne motion models, which accurately can describe apparent scene motion caused by translations and rotations of the camera and remote scenes.

Since this accurate motion estimation already is performed, other techniques such as electronic stabilization and mosaic generation can be implemented without much problems. As a matter of fact, the standard output of DSR already is stabilized, and mosaic generation is available as a standard option. Figure 3 shows an example of a mosaic generated from an air-to-ground image sequence, which contains a road, houses, and trees.

\section{MOVING TARGET DETECTION}

One implicit output of the DSR algorithm is a cue for scene change. Under the assumption of a slowly varying scene, only small scene changes per scene can be expected. However, in the presence of moving targets these scene changes will be signifcant at the locations of these targets. This allows these scene changes to be used as moving target indicators, which together with a proper tracking algorithm can be used for Moving Target Detection (MTD).

Obviously, random motion patterns by moving objects cannot be modeled by the generic motion models used in SBME. Whenever moving objects are large and/or high-contrast, they will adversely contribute to SBME's accuracy. However, since moving object cues already are available by the DSR algorithm, identi£cation of static scene elements is not dif£cult. Subsequently, the static scene elements can be used as SBME inputs, leading to an undisturbed motion estimate. An example of this can be seen in £gure 4. 


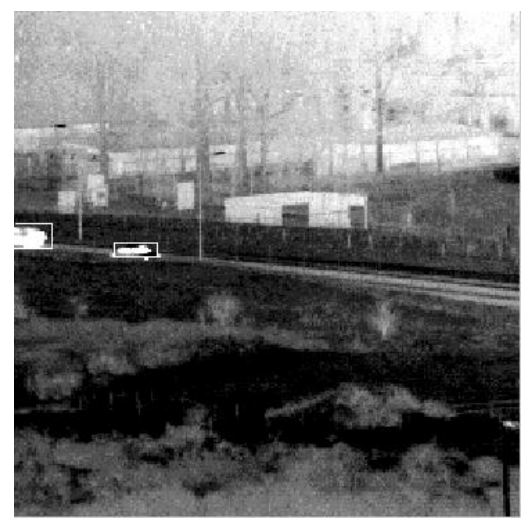

(a)

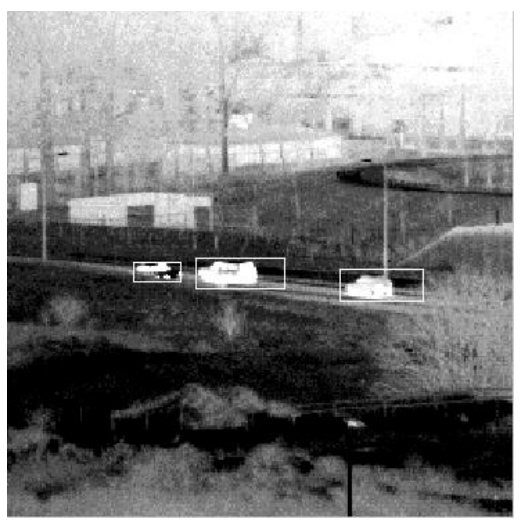

(b)

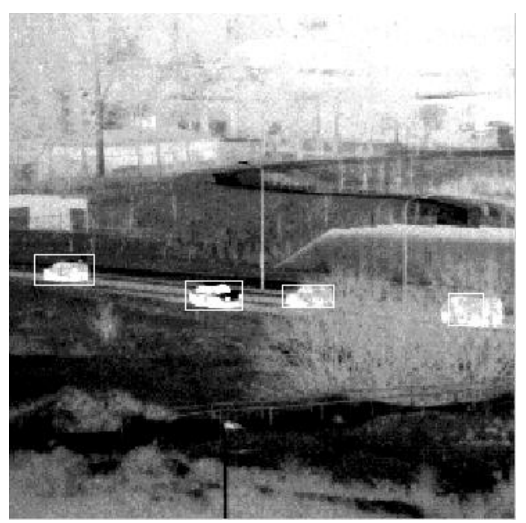

(c)

Figure 4. Moving target detection with moving scene and moving objects. Detections are visualized over the DSR result image for selected frames in the sequence.

\section{A COMBINED ALGORITHM SET}

The previous sections have described the SBNUC, DSR, SBME and MTD algorithms. As the Enal output quality of the separate functions all critically depend on the quality of their input data, care has to be taken that also this input data is of high quality. We have implemented a fully integrated solution of these functionalities, as this integration allows that all algorithms can beneft from the signal conditioning capabilities of the other algorithms. As such this integration guarantees that optimal overall performance will be reached.

This combination of algorithms also allows implementation of specifc applications without signifcant extra computational effort, such as for example MTD in a step-stare generated mosaic, allowing surveillance of a large feld of regard in a single display.

Another beneft of an integrated algorithm set is that intermediate results are made available between the algorithms, eliminating re-calculations, and thus reducing the overall computational needs.

Based on the considerations given above, it is our view that the best way to provide these functionalities is by combining them into an integrated algorithm set. Only then optimal performance is guaranteed as with separate application the algorithms cannot utilize on the signal conditioning capabilities of the other algorithms.

\section{CONTRAST ENHANCEMENT}

The application of an advanced signal conditioning set as presented above will result in high dynamic images imagery with an excellent signal to noise ratio. The dynamic range of such imagery will easily exceed the display capabilities of standard display devices, leading to a loss of information in the visualization process.

To allow visualization of all information present in the high-quality imagery we propose the use of Local Adaptive Contrast Enhancement (LACE) LACE is an algorithm to compress the overall dynamic range of the images while retaining small amplitude details and maintaining an overall natural look. ${ }^{7}$ As depicted in fgure 5, human observer experiments have reported signifcant improvement of operator tasks after application of LACE. ${ }^{6}$

\section{EXAMPLES}

The algorithms described above are applied to a wide set of different scenarios and cameras. Common to the examples given in this paper is that they all report on application of the algorithms against an image sequence with camera-to-scene motion.

In £gure 1 results are shown for the performance of SBNUC. It depicts a part of Scheveningen near TNO-FEL. In image 1(a) no standard NUC has been applied. Application of SBNUC results after only 10 frames already in the cleanedup image shown in 1(b). All point-like non uniformities are removed but large structures remain. Image 1(c) shows that, after having processed more frames, SBNUC also will remove these larger structures.

Proc. of SPIE Vol. 5076 


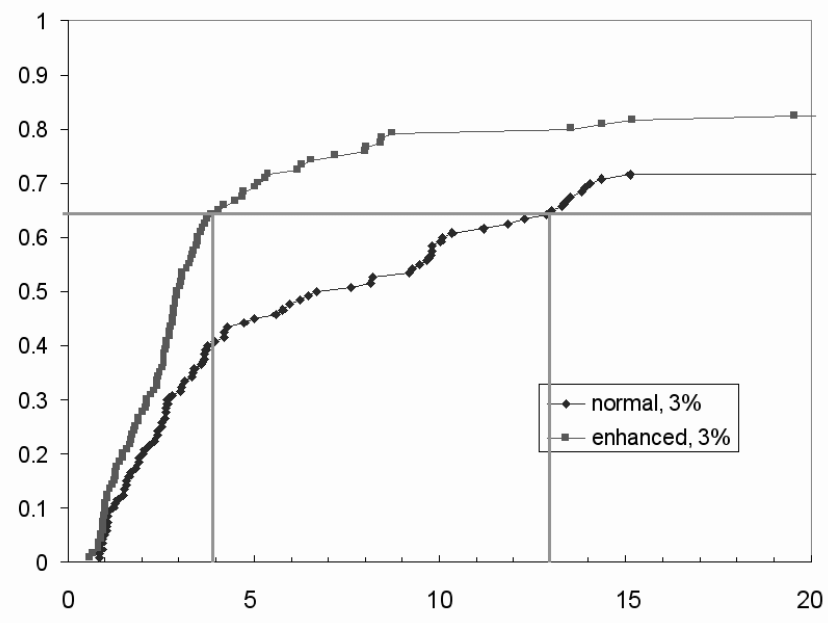

Figure 5. Performance of LACE. Shown is the cumulative probability of detection as function of search time. The task is the detection of artifcial moving point targets in a MW IR air to ground scenario. The use of LACE decreased the search time for $65 \%$ of all targets from 13 to 4 seconds.

An example of mosaicking is given in £gure 3. From single images in the sequence it is impossible to determine the surrounding of the current £eld of regard, seriously limiting the situational awareness of the system's operator. Application of the mosaicking process retains all details of the individual images while providing the overview to, for example, access the relation between the road and the town as shown in the fgure.

The moving target capabilities are illustrates in £gure 4. This £gure shows the result of moving target detection on a mid-wave IR sequence. The sequence depicts moving cars, and at the same time the camera is panning over the scene. The results show that the moving cars are properly detected.

Figure 6 shows application of DSR-4 to a long-range MW IR image. The upper part shown is a $80 \times 40$ region of interest of an original image. The lower part depicts the result of 4 times resolution enhancement. Here application of DSR enables the identifcation of the overall coastline structure, as well as providing details in the structure and aoors in the buildings enabling recognition of these buildings.

Figure 7 depicts the results of applying DSR-2 and LACE to an image of an LW uncooled handheld IR imager. The scene had a good contrast, and the standard non-uniformity correction procedure had just been applied, resulting in a relatively noise-free original image. Application of both DSR-2 and LACE enables better identifcation of details in the vehicles, such as the windshield wipers, mirrors, and antennas.

Figure 8 shows results of the same handheld IR camera. Here the same non-uniformity correction had been used as in £gure 7. Due to a signifcant difference in average (apparent) temperature between the scenes in the fgures 8 and 7, this resulted in the extreme non-uniformities in £gure 8. However, application of SBNUC dramatically increases this image quality. Application of temporal noise reduction removes the temporal noise inherent to a low-end handheld IR imager and longer ranges. Finally, the application of LACE to this image enables visualization of all information in the image.

In £gure 9 results are shown for a gen-III image intensifer. The original image is disturbed by noise and interlacing. DSR will decrease the noise while increasing the defnition of the image, as can be seen with the person walking, the trees in the foreground and the fence in the background.

The noise reduction capabilities of DSR are highlighted in £gure 10. Here the noise present in CCD images acquired in low-light conditions is drastically reduced.

\section{CONCLUSIONS}

The application of signal conditioning algorithm techniques can lead to dramatic increase of the performance of imaging systems. The paper presented SBNUC for non uniformity correction, DSR for noise reduction, resolution enhancement 


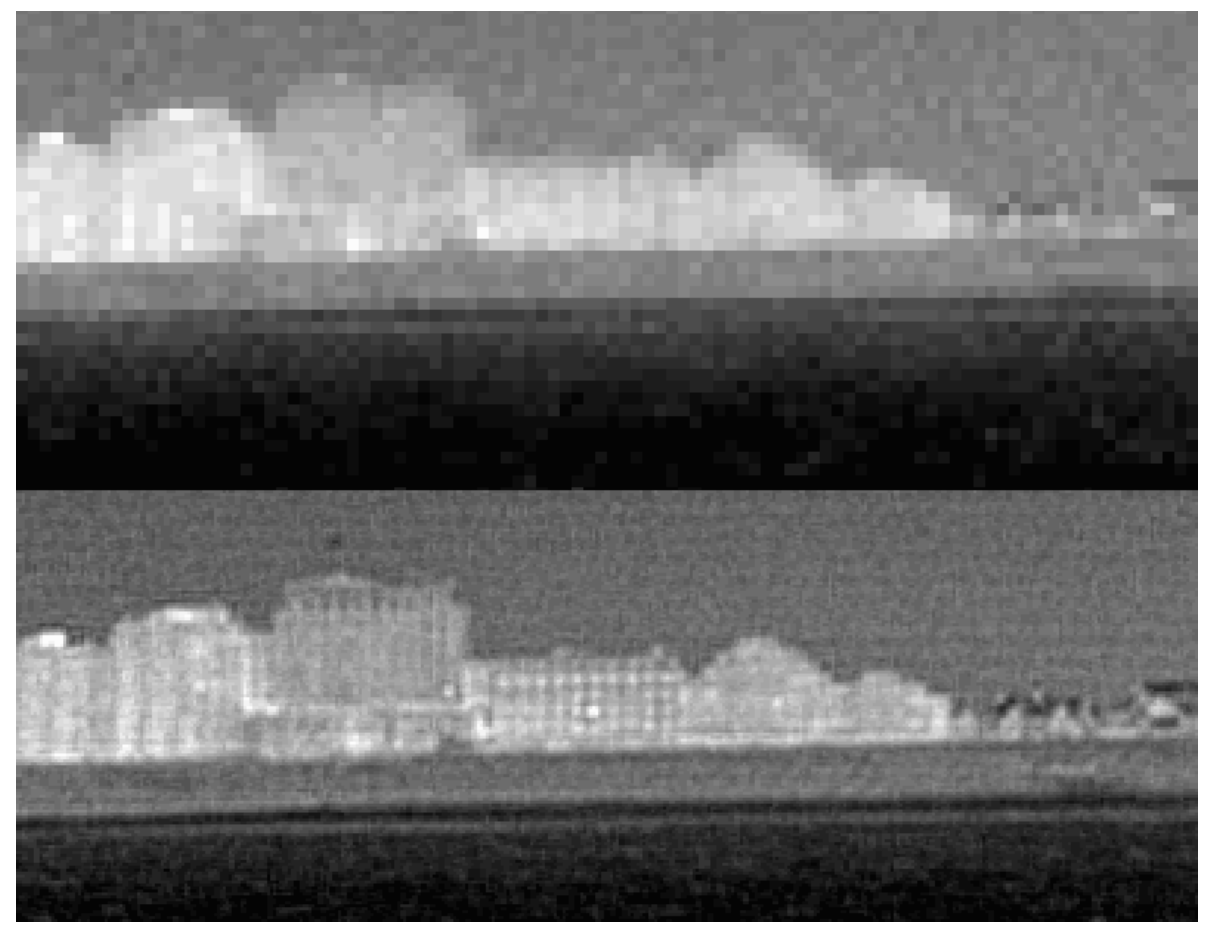

Figure 6. Top: part of original MW IR image, depicting the sea-side town of Noordwijk over a distance of six nautical miles. Bottom: DSR-4 result of this image.

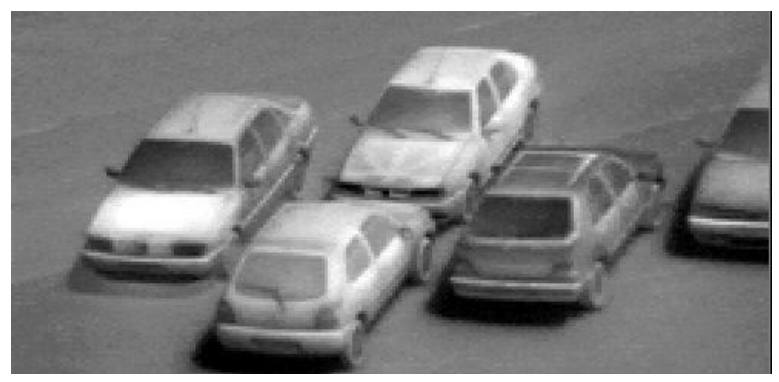

(a)

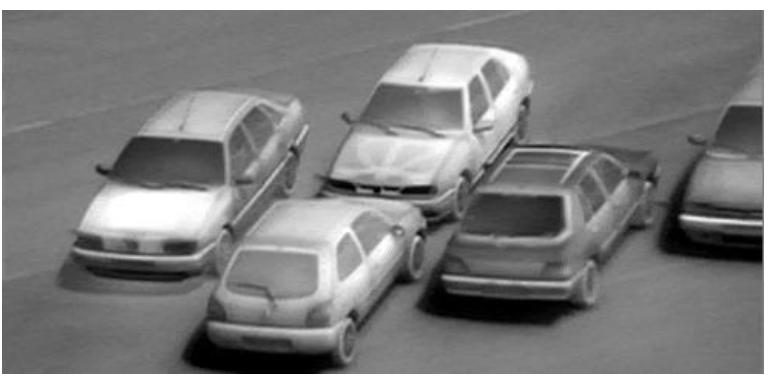

(b)

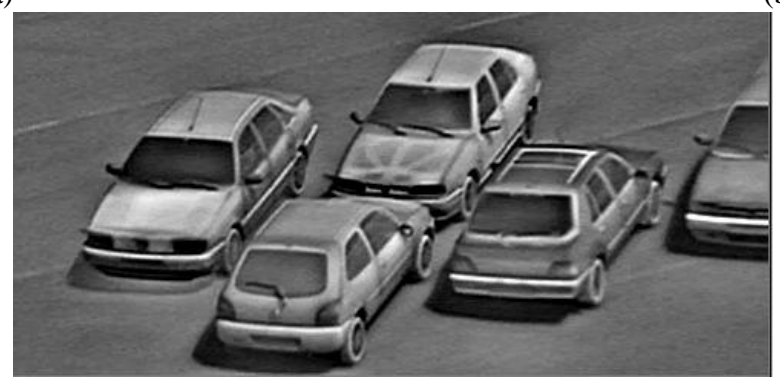

(c)

Figure 7. Results for high-contrast LW uncooled IR imagery. (a) Original image, just after calibration procedure. (b) Image after application of DSR-2 processing. (c) Image after DSR-2 and LACE processing. 


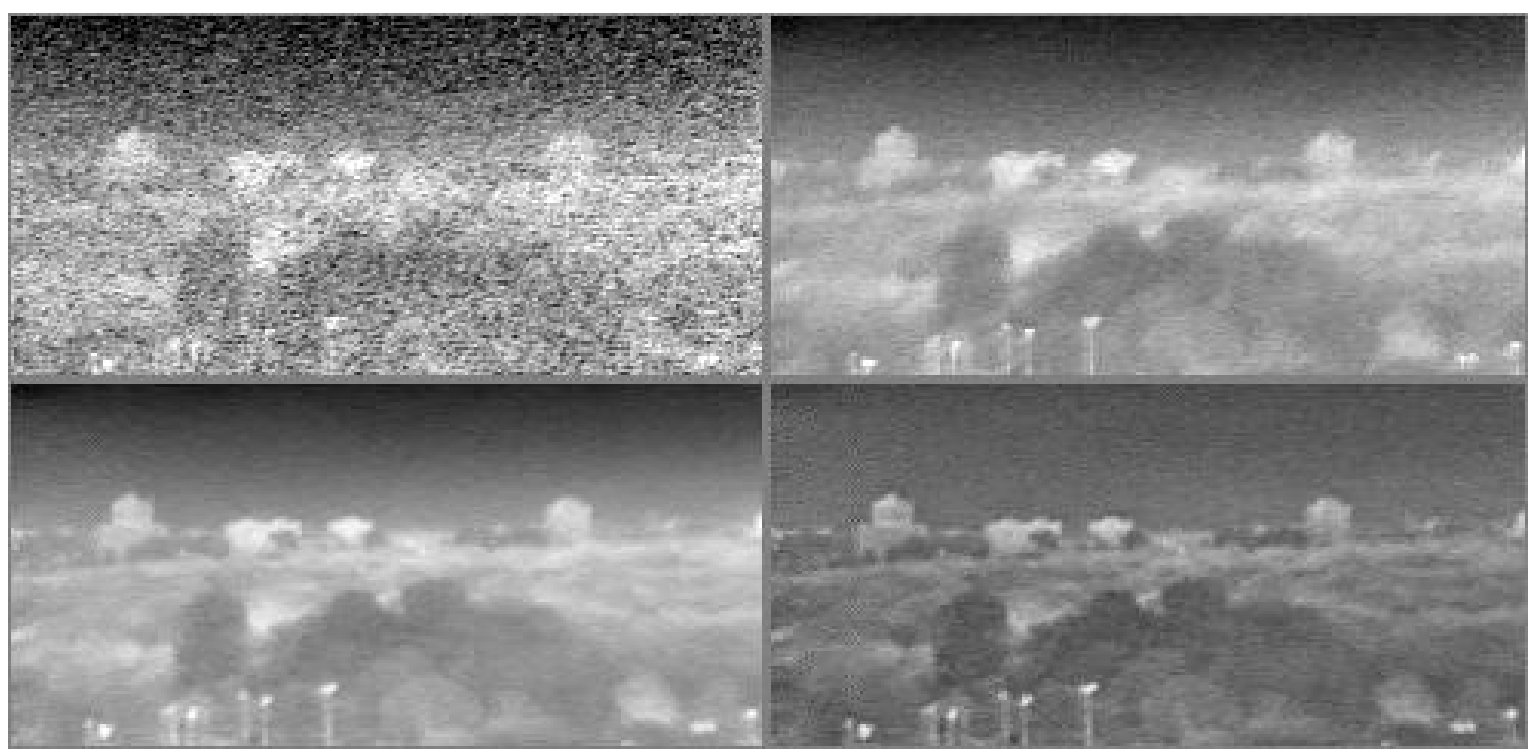

Figure 8. Results for low-contrast LW uncooled IR imagery. Top-left: original image. Top-right: image after SBNUC. Bottom-left: image after SBNUC and temporal noise reduction. Bottom-right: image after SBNUC, temporal noise reduction, and LACE.

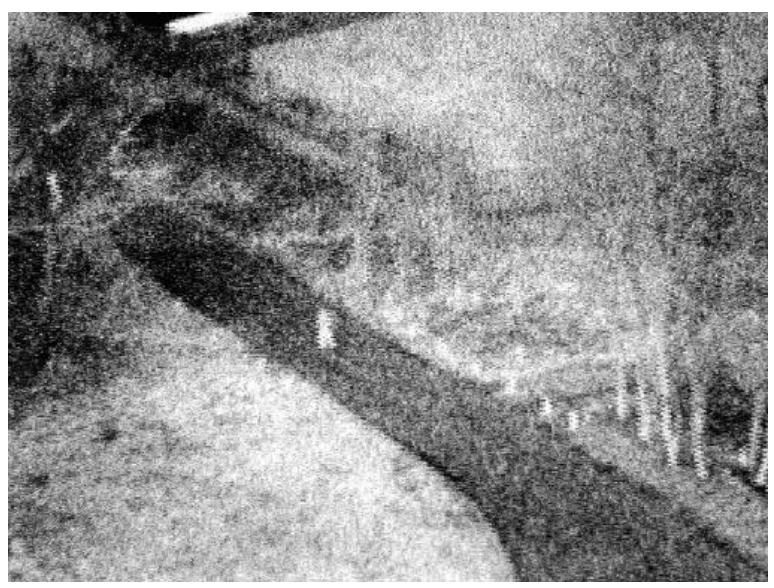

(a)

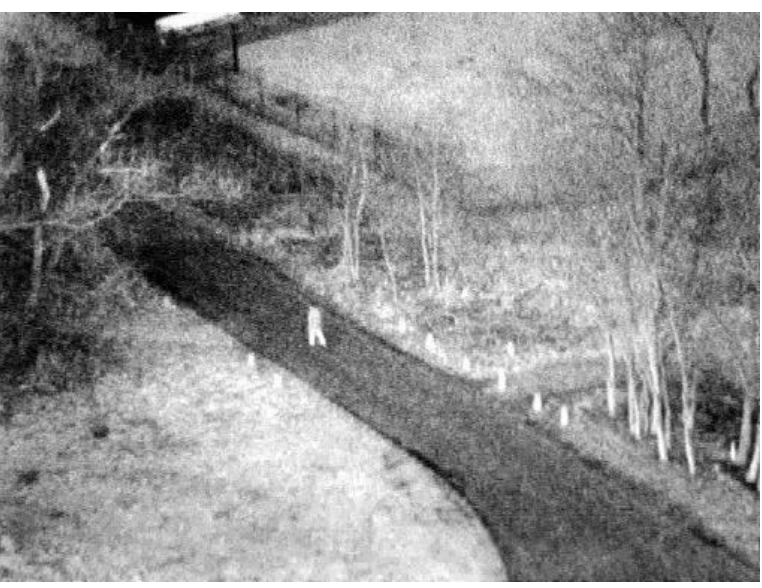

(b)

Figure 9. Results for Image Intensifed imagery. (a) Original image. (b) Original image after temporal noise reduction. 


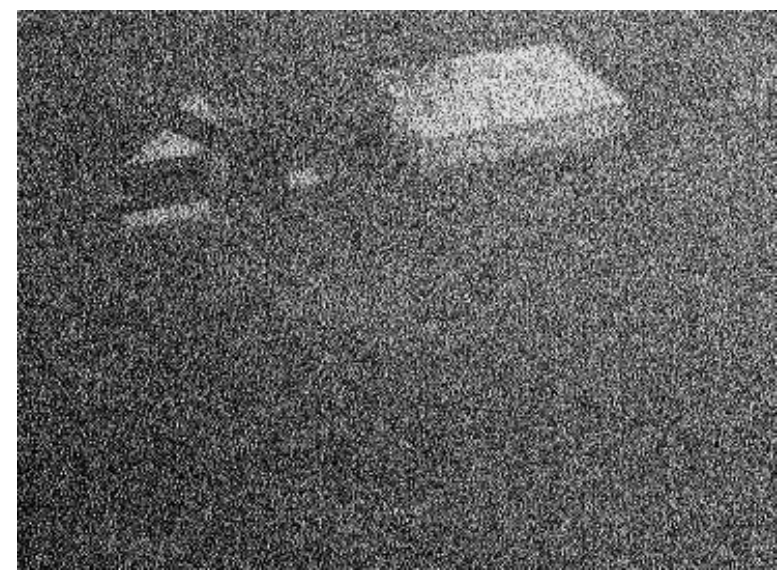

(a)

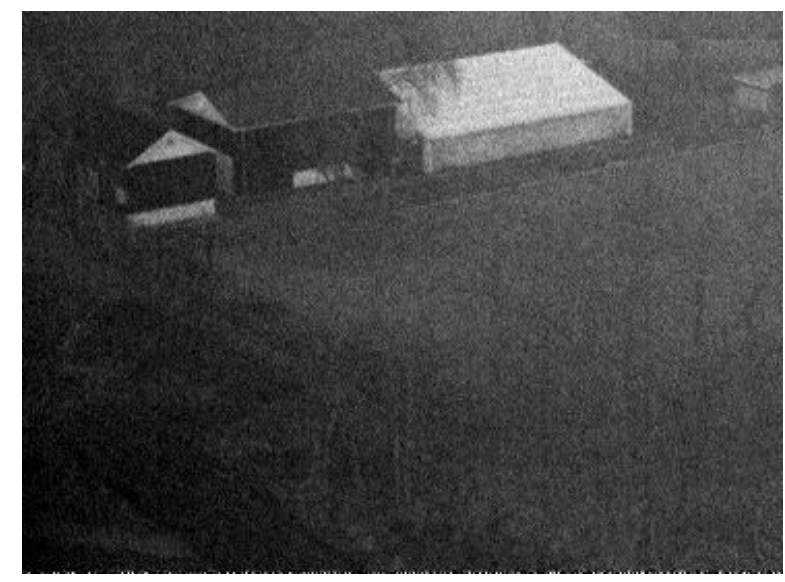

(b)

Figure 10. Results for low-light CCD imagery. (a) Original image. (b) Temporal noise reduced result.

and increased sharpness, MTD for target detection, LACE for optimal visualization as well as mosaicking of an image sequence. For different scenarios and camera systems signifcant improvement in the image quality is demonstrated.

\section{REFERENCES}

1. M. S. Alam, J. G. Bognar, R. C. Hardie, and B. J. Yasuda. High resolution infrared image reconstruction using multiple, randomly shifted, low resolution, aliased frames. In Proc. SPIE Vol. 3063, Infrared Imaging Systems: Design, Analysis, Modeling, and Testing VIII, 1997.

2. M.-C. Chiang and T. Boult. Ef£cient super-resolution via image warping. Image and Vison Computing, 18:761-771, 2000.

3. R. C. Gonzalez and P. Wintz. Digital Image Processing. Addison-Wesley Publishing Company, 1997.

4. R. C. Hardie, K. J. Barnard, and E. E. Armstrong. Joint MAP registration and high-resolution image estimation using a sequence of undersampled images. IEEE Trans. on Image Proc., 6:1621-1633, 1997.

5. C. L. Hendriks and L. van Vliet. Improving resolution to reduce aliasing in an undersampled image sequence. In Proc. SPIE, Vol. 3965, Sensors and Camera Systems for Scientifc, Industrial and Digital Photography Applications, 2000.

6. M. A. Hogervorst and P. Bijl. Validation of the FEL image enhancement algorithm. Technical Report TM-00-C039, TNO Human Factors, Oct. 2000.

7. K. Schutte. Multiscale adaptive gain control of IR images. In B. F. Andresen and M. S. Scholl, editors, Proc. SPIE Vol. 3061, Infrared Technology and Applications XXIII, pages 906-914, Orlando (FL), USA, Apr. 1997.

8. G. Wolberg. Digital Image Warping. IEEE Computer Society Press, 1990. 